\title{
Mean-Field and Anomalous Behavior on a Small-World Network
}

\author{
M. B. Hastings \\ Center for Nonlinear Studies and Theoretical Division, Los Alamos National Laboratory, Los Alamos, NM 87545, \\ hastings@cnls.lanl.gov
}

(April 21, 2003)

\begin{abstract}
We use scaling results to identify the crossover to mean-field behavior of equilibrium statistical mechanics models on a variant of the small world network. The results are generalizable to a widerange of equilibrium systems. Anomalous scaling is found in the width of the mean-field region, as well as in the mean-field amplitudes. Finally, we consider non-equilibrium processes.
\end{abstract}

The appropriate description for many complex realworld systems is as a network [1], a general connection of nodes and vertices which need not have the structure of a regular lattice. The small-world network model combines both long-range and short-range aspects, and interpolates between regular lattices and random graphs. This model [2], in which a regular lattice is modified by either randomly "re-wiring" links or else by simply randomly adding long-range links, has become a standard model of real-world networks. It incorporates some notion of locality, as most of the links remain the same as that of the original underlying lattice. Yet it also includes the "small world effect", that the average path length between sites on the network scales only as the logarithm of the network size.

Cooperative behavior on networks is a major topic of interest. Studies of equilibrium statistical on these systems have shown rich behavior $[3-6]$. Work $[7,8]$ on networks with long tails in the probability distribution of the coordination number has found alternatives to mean-field behavior. We will instead study the behavior on smallworld systems. The technique will be generically applicable to a wide-range of problems. We show the existence of mean-field behavior, albeit with anomalous exponents describing the width of the critical region and various mean-field amplitudes. These anomalous exponents can complicate the interpretation of numerical data. Finally, we will consider a non-equilibrium case, describing the relaxation to a stationary state via a branching process.

The presence of quenched randomness makes the small-world model difficult to treat analytically. We instead consider a different, but related model, which lacks quenched randomness, considering a variety of equilibrium and non-equilibrium models. We again start with a regular lattice of $V$ sites in $d$ dimensions. Rather than adding long-range links with probability $p$, we give each site of the lattice a weak coupling, of order $p / V$, to every other site in the lattice [9]. We will refer to this as the long-range model. We will find that many results can be obtained on this system by combining mean-field with standard renormalization group techniques. Later, we will compare this model to the standard small world model; we will argue that in many cases the presence of quenched randomness in the small world model is irrelevant, and thus scaling results for the small world model can be obtained from the long-range model. It is interesting to compare to a related mean-field solution of path lengths on a small work network [10]. Finally, we will show the long-range model provides an upper bound for the free energy of the small world network model.

In some cases, the long-range model may also be more appropriate than the usual small world model. In the spread of a disease, for example, people tend to spread the disease to those geographically nearby (the regular lattice). There is a chance of a long-range spread of the disease. However, this is not necessarily due to fixed longrange links. Rather, it is due to the random probability that a given person travels a long distance, typically by air. Thus, a slight probability of long-range contact between any two people may be a better description than a set of fixed long-range links.

Equilibrium Statistics - We consider any equilibrium statistical mechanics model, such as an Ising model, XY model, etc... These models can be represented by introducing a field $\phi(x)$, where $x$ labels lattice sites and where $\phi$ has $1,2, \ldots$ components, with a partition function

$$
Z=\sum_{\{\phi\}} \exp [-S[\phi]]
$$

where $S=E / k T$ is a statistical weight for a configuration of energy $E$ at temperature $T$.

For a model on a regular $d$-dimensional lattice, $S[\phi]=$ $S_{\text {local }}[\phi]$, a local function of $\phi$. We refer to this as the local system. We choose instead for the long-range model a statistical weight with additional long-range couplings of strength $p / V$ :

$$
S[\phi]=S_{\text {local }}[\phi]-\frac{p}{2 V} \sum_{x_{1}, x_{2}} \phi\left(x_{1}\right) \cdot \phi\left(x_{2}\right) .
$$

Then, decouple the long-range interaction to find

$$
Z=\int_{-\infty}^{\infty} \mathrm{d} h \exp \left[-\frac{V h^{2}}{2 p}\right] Z(h)
$$

where

$$
Z(h)=\sum_{\{\phi\}} \exp \left[-S_{\text {local }}[\phi]+h \sum_{x} \phi x\right] .
$$


Here, $Z(h)$ is equal to the partition function of the local system in the presence of a magnetic field, $h$.

For $p$ large, the long-range interaction outweighs the short-range interaction, and the system can be approximately solved by mean-field theory. We will instead consider precisely the opposite case: when $p$ is small. Then, the critical point of the long-range system, $\tilde{T}_{c}$, is close to the critical point of the local system, $T_{c}$. Thus, we can use scaling laws for the local system: the magnetization, $m$, obeys $m\left(T=T_{c}, h\right)=A_{m}|h|^{1 / \delta}$, defining the critical exponent $\delta$. For $T>T_{c}$, the susceptibility $\chi$ obeys $\chi(T, h=0)=A_{\chi}^{+}\left|T-T_{c}\right|^{-\gamma}$. In general, we can write a scaling function: $m=h^{1 / \delta} f\left(\left(T-T_{c}\right) h^{-1 /(\delta \beta)}\right)$. For $h>>\left(T-T_{c}\right)^{\delta \beta}$, we use the first result $m\left(T=T_{c}, h\right)=$ $A_{m}|h|^{1 / \delta}$, while for $h<<\left(T-T_{c}\right)^{\delta \beta}$, we use the second $m\left(T=T_{c}, h\right)=A_{\chi}^{+} h\left|T-T_{c}\right|^{-\gamma}-B_{\chi} h^{3}\left|T-T_{c}\right|^{-\gamma-2 \delta \beta}$, where we have added the $h^{3}$ term in the expansion of $m$.

The magnetization is defined by $\partial \ln Z(h) / \partial h=m V$. Thus,

$$
Z(h)=Z(0) \exp \left[\int_{0}^{h} \mathrm{~d} h^{\prime} m\left(h^{\prime}\right) V\right]
$$

We start by considering the case of $T$ near $T_{c}$ so that $h>>\left(T-T_{c}\right)^{\delta \beta}$. Then, Eqs. $(3,5)$ give

$$
Z=Z(0) \int_{-\infty}^{\infty} \mathrm{d} h \exp \left[-\frac{V h^{2}}{2 p}+A_{m} V h^{1+1 / \delta} /(1+1 / \delta)\right]
$$

Since $V$ is taken large, we can use a saddle point to arrive at $h=\left(p A_{m}\right)^{\delta /(\delta-1)}$, or

$$
m=A_{m}\left(p A_{m}\right)^{1 /(\delta-1)} .
$$

The correlation length $\xi$ of the local system in the presence of this field is proportional to $m^{-\nu / \beta}$, and hence diverges as $p \rightarrow 0$. The meaning of the correlation length is not that spins beyond this length are uncorrelated. Rather, it is that beyond this length the correlations in the long-range system are controlled by the average field $m$, while below this length, the fluctuations are important. Thus, the correlation function $\langle\phi(0) \phi(x)\rangle$ decays as a power law up to the correlation length, and then asymptotes to a constant.

We have seen that at $T=T_{c}$, the system has a net magnetization, and thus $T_{c}<\tilde{T}_{c}$. To study the transition itself, we now consider the second case, $h<<\left(T-T_{c}\right)^{\delta \beta}$. Now, Eqs. $(3,5)$ give

$$
\begin{array}{r}
Z=Z(0) \int_{-\infty}^{\infty} \mathrm{d} h \exp \left[-\frac{V h^{2}}{2 p}+A_{\chi}^{+} V h^{2}\left|T-T_{c}\right|^{-\gamma} / 2\right. \\
\left.-B_{\chi} V h^{4}\left|T-T_{c}\right|^{-\gamma-2 \delta \beta} / 4\right] .
\end{array}
$$

Again using a saddle point, we find a critical point at $\tilde{T}_{c}$ given by

$$
\tilde{T}_{c}-T_{c}=\left(p A_{\chi}^{+}\right)^{1 / \gamma}
$$

with $m=0$ for $T>\tilde{T}_{c}$. Slightly below the critical point we find $h=\sqrt{\tilde{T}_{c}-T} \sqrt{\frac{\gamma A_{\chi}^{+}}{B_{\chi}}}\left(\tilde{T}_{c}-T_{c}\right)^{\delta \beta-1 / 2}$, and a magnetization given by

$$
\begin{aligned}
m= & \sqrt{\tilde{T}_{c}-T} A_{\chi}^{+} \sqrt{\frac{\gamma A_{\chi}^{+}}{B_{\chi}}}\left(\tilde{T}_{c}-T_{c}\right)^{\delta \beta-1 / 2-\gamma} \\
& =\sqrt{\tilde{T}_{c}-T} A_{\chi}^{+} \sqrt{\frac{\gamma A_{\chi}^{+}}{B_{\chi}}}\left(\tilde{T}_{c}-T_{c}\right)^{\beta-1 / 2} .
\end{aligned}
$$

Thus, the magnetization behaves as $m=\tilde{A} \sqrt{\tilde{T}_{c}-T}$, with $\tilde{A} \propto p^{(\beta-1 / 2) / \gamma}$. If the local system is described by mean-field theory, then $\beta-1 / 2=0$. In other cases, $\beta<1 / 2$, and the mean-field amplitude $\tilde{A}$ diverges for small $p$. From Eq. (8), the specific heat jump at $T=\tilde{T}_{c}$ is equal to $\left(\gamma A_{\chi}^{+}\left|\tilde{T}_{c}-T_{c}\right|^{-\gamma-1}\right)^{2} /\left(2 B_{\chi}\left|\tilde{T}_{c}-T_{c}\right|^{-\gamma-2 \delta \beta}\right) \propto$ $\left|\tilde{T}_{c}-T_{c}\right|^{2 \delta \beta-\gamma-2}=\left|\tilde{T}_{c}-T_{c}\right|^{-\alpha}$. Thus, the jump in specific heat at the mean-field transition is of order the specific heat of the local system at temperature $\tilde{T}_{c}$.

We now consider the width of the mean-field critical region. If we extrapolate Eq. (10) to $T=T_{c}$, we find that the power of $p$ in the result is consistent with the power of $p$ in Eq. (7). For $T<T_{c}$, the average magnetization of the local system in the absence of a field behaves as $\left|T_{c}-T\right|^{\beta}$, and the susceptibility is given by $\chi=A_{\chi}^{-}\left|T_{c}-T\right|^{-\gamma}$. In the long-range system, this magnetization produces a field $h \propto p\left|T_{c}-T\right|^{\beta}$, which in turns feeds back and increases the magnetization an amount of order $p\left|T_{c}-T\right|^{\beta}\left|T_{c}-T\right|^{-\gamma}$. For $\left|T_{c}-T\right|^{\gamma}>>p$, this effect is negligible compared to the averaged field itself, $\left|T_{c}-T\right|^{\beta}$. Thus, at such temperatures the long-range interactions have negligible effect on the magnetization and so the mean-field critical behavior only extends to $\left|T_{c}-T\right| \propto p^{1 / \gamma} \propto\left|\tilde{T}_{c}-T_{c}\right|$. Therefore, for small $p$, the width of the mean-field critical region is small. The scaling arguments above all rely on this width becoming narrower than the width of the anomalous critical region in the local system, in which case both mean-field and anomalous scaling will be seen in the same system.

Effect of Randomness- We now consider the difference between the long-range model, which lacks randomness and has links of strength $p / V$, and a version of the smallworld model in which the local network is modified by adding strong links connecting sites with a probability $p / V$. The strategy is to consider the system without randomness, and to consider the effects of randomness as a perturbation. We verify that it is self-consistent to ignore randomness, at least for small $p$.

At $\tilde{T}_{c}$, the correlation length of the local system is $\xi \propto$ $\left(\tilde{T}_{c}-T\right)^{-\nu} \propto p^{-\nu / \gamma}$. Thus, within a correlation volume, 
there are $p^{-\nu d / \gamma}$ sites. The correlation volume can be characterized by the average field acting on it, and the average temperature of the correlation volume.

First consider the average field. If sites in the correlation volume are chosen instead with probability $p$ to have long-range links, then an average of $p^{1-\nu d / \gamma}$ sites are chosen. Since $1-\nu d / \gamma<0$, this number diverges as $p \rightarrow 0$. Then, there are a large number of such sites within each correlation volume and so the fluctuation in the number of such sites within each correlation volume is negligible. Thus, the fluctuation in the average field is negligible.

Now, consider the average temperature. A site that is coupled via a long-range link has reduced correlations with its neighbors compared to one which does not have such a link. The site it is coupled to via the long-range link produces an average field proportional to $m$, and also produces fluctuations about this field. Thus, effectively the temperature of a site with a long-range link is raised, as the fluctuations in the field reduce its correlations with its neighbors. Consider the number of sites with long-range links. The root mean square fluctuation in the number of such sites scales as $\left(p \xi^{d}\right)^{1 / 2}$, and thus the fluctuation in the temperature averaged over a correlation volume scales as $\left(p / \xi^{d}\right)^{1 / 2} \propto p^{1 / 2+\nu d /(2 \gamma)} \propto$ $\left|\tilde{T}_{c}-T_{c}\right|^{\gamma / 2-\nu d / 2}$. Compare this to the difference in temperatures, $\tilde{T}_{c}-T_{c}$. As long as

$$
\gamma / 2+\nu d / 2>1
$$

the fluctuation in temperature is also negligible. Eq. (11) resembles the Harris criterion [11] for the relevance of disorder, with an additional term $\gamma / 2$ on the left-hand side. This additional term guarantees that the inequality holds for all models that we know. Thus, fluctuations in field and temperature are both negligible and the scaling of both $\tilde{T}_{c}-T$ and $\tilde{A}$ with $p$ should be the same in the small-world and long-range models.

Variational Approach - In addition to the scaling arguments above, we now show that the long-range model without randomness provides an upper bound to the free energy of a small world network model, using an argument inspired by the Migdal-Kadanoff bond-moving procedure [12]. Eq. (2) defines the statistical weight $S$ without randomness. If instead of connecting every pair of sites with strength $p / V$, we add connections between pairs of sites with probability $p / V$ and unit strength, we obtain a new statistical weight

$$
\tilde{S}[\phi]=S_{\text {local }}[\phi]-\frac{1}{2} \sum_{x_{1}, x_{2}} \phi\left(x_{1}\right) \cdot \phi\left(x_{2}\right),
$$

where the sum extends over sites $x_{1}, x_{2}$ which are connected by a long range link. Then, $\langle S-\tilde{S}\rangle=$ $\sum_{x_{1}, x_{2}} w\left(x_{1}, x_{2}\right)\left\langle\phi\left(x_{1}\right) \phi\left(x_{2}\right)\right\rangle$, where the brackets \langle\rangle denote an expectation value computed with statistical weight $e^{-S}$, and where $w\left(x_{1}, x_{2}\right)=-p /(2 V)$ if $x_{1}, x_{2}$ are not connected in the small world network and $w\left(x_{1}, x_{2}\right)=1-p /(2 V)$ if $x_{1}, x_{2}$ are connected. The quantity $\langle S-\tilde{S}\rangle$ is a random function of disorder. However, for a large system, this quantity is self-averaging. If pairs are connected with probability $p / V$, the average over disorder, $\overline{\langle S-\tilde{S}\rangle}$, is equal to zero. Thus, for typical networks, $\langle S-\tilde{S}\rangle=0$, up to small fluctuations, and thus by a convex inequality, $\tilde{Z}_{\text {typ }} \geq Z$, where $\tilde{Z}_{\text {typ }}$ is the partition function for a typical small world network, with statistical weight $e^{-\tilde{S}}$.

This shows that the free energy of such a small world model is less than or equal to the free energy of the longrange model. Intuitively, we expect that the transition temperature in the long-range model will be higher than that in the small-world model: if we ignore the local couplings, and consider only the long-range links then this statement is definitely true. Above we have argued that $\tilde{T}_{c}-T$ should scale as the same power of $p$ in both the small-world and long-range models. Even if that argument were to break down, we should expect that $\tilde{T}_{c}-T_{c}$ scales as at least as large a power of $p$ in the small-world model as it does in the long-range model.

Comparison to Numerics - An important work was a numerical calculation of some of these quantities, looking for the shift in the transition temperature [6]. In that paper, a different scaling argument was made for the shift, $\tilde{T}_{c}-T_{c} \sim p^{1 /(\nu d)}$. This is the temperature at which a correlation volume includes roughly one long-range link. However, we have argued that the shift in transition temperature actually scales as $p^{1 / \gamma}$, which is less than $p^{1 /(\nu d)}$ as $p \rightarrow 0$. The difference arises since one long-range link is not sufficient to magnetize an entire correlation volume; several such links are required.

The numerical results in two dimensions are consistent with a shift in transition temperature scaling as $p^{1 / \gamma}=p^{0.57 \ldots}$. The numerical results in three dimensions indicate a shift scaling as $p^{0.96}$, while taking $\gamma=1.2396$ from $\epsilon$-expansion [13] gives $1 / \gamma \approx 0.81$. This indicates some discrepancy with the numerical results. However, in the numerical study [6], it was argued that their results do not yet involve sufficiently large lattices to obtain accurate scaling; certainly, $p^{0.81}$ is closer to the observed scaling than $p^{1 /(\nu d)} \approx p^{0.53}$ is.

Non-Equilibrium Dynamics - We now consider the generalization to a non-equilibrium process, the contact process [14], in which each site is marked either infected or susceptible. An infected site becomes susceptible at unit rate, while an infected site can turn a neighboring susceptible site infected at a rate $\lambda / q$, with $q$ the lattice coordination number. The state with all sites susceptible is absorbing. However, above a critical $\lambda_{c}$, if a single infected site is placed in an infinite lattice of susceptible sites, there is a non-zero probability of the epidemic persisting for all time. We modify the model as follows: each susceptible site can be infected by any other infected site, 
not necessarily a neighbor, at a rate equal to $p / V$. Although we will not decouple this interaction, the general development will be very similar to the equilibrium case.

We start by recalling some exponents in the local case. For $\lambda>\lambda_{c}$, there is an average density, $\rho \propto\left|\lambda-\lambda_{c}\right|^{\beta}$. In the presence of a source, where susceptible sites become infected at a rate $h$, the density $\rho\left(\lambda=\lambda_{c}, h\right)=A_{\rho} h^{1 / \delta_{h}}$.

Consider also the infection spreading from a single source. At $\lambda=\lambda_{c}$, the survival probability of the infection after time $t, P(t)$ obeys $P(t)=A_{p} t^{-\delta}$, The number of infected sites is a random variable, $n(t)$; the average number of such sites obeys $\bar{n}(t)=A_{n} t^{\eta}$. The radius of the infection scales as $t^{z / 2}$. For $\lambda<\lambda_{c}$, the infection dies out exponentially, with an asymptotic survival probability $P(t) \propto e^{-t / \tau}$, with $\tau \propto\left|\lambda_{c}-\lambda\right|^{-\nu_{\|}}$. This gives rise to a divergent susceptibility: in the presence of a source $h$, the susceptibility, $\chi \equiv \partial_{h} \rho$, obeys $\chi(\lambda, h=0)=A_{\chi}\left|\lambda_{c}-\lambda\right|^{-\gamma}$.

Now, consider the dynamics in the long-range model, with $\lambda=\lambda_{c}$, with a single source for an infection. This source grows as described, with the given $P(t), \bar{n}(t)$. However, the local outbreak starting from that source can produce other local outbreaks elsewhere, via the longrange links, at a rate equal to $p$ times the number of infected sites. For $p$ small, the number of infected sites $n(t)$ will be large before such an event, and thus the fluctuations in the $n(t)$ are described by a random process with a universal distribution. In the large $V$ limit, at fixed $t$, each new local outbreak produced via a long-range link is well separated in space from the other local outbreaks. Thus, we can describe the dynamics of the spread from a single source simply: there is initially one local outbreak, created at time 0 , which survives at time $t$ with probability $P(t)$, and which produces additional local outbreaks at a rate equal to $p n(t)$. Each local outbreak, created at time $t^{\prime}$, evolves independently, surviving with probability $P\left(t-t^{\prime}\right)$, and producing additional local outbreaks with rate $p n\left(t-t^{\prime}\right)$. This fully describes the dynamics via a branching process. For $\lambda \neq \lambda_{c}$, this description of the dynamics remains valid with a changed $P(t)$ and distribution of $n(t)$.

At short times, the average number of infected sites in this dynamics is equal to $\bar{n}(t)$. At long times, the average number of infected sites grows exponentially. To describe this exponential growth, realize that at long times the number of local outbreaks becomes large. If $s\left(t^{\prime}\right)$ describes the number of local outbreaks started at time $t^{\prime}$, then the average number of particles at time $t$ is equal to $\int_{0}^{t} \mathrm{~d} t^{\prime} s\left(t^{\prime}\right) n\left(t-t^{\prime}\right)$, and thus on average $s(t)=$ $p \int_{0}^{t} \mathrm{~d} t^{\prime} s\left(t^{\prime}\right) n\left(t-t^{\prime}\right)$. Inserting an ansatz $s(t)=e^{\alpha t}$, we find that

$$
\alpha=\left[A_{\eta} p \Gamma(1+\eta)\right]^{1 /(1+\eta)} .
$$

Each local outbreak takes a volume of order $t^{d z / 2}$. Eventually, at sufficiently large time, such that $e^{\alpha t} \sim$
$\left(V / t^{d z / 2}\right)$, the individual local outbreaks start to merge, and the dynamics of different local outbreaks become coupled. This time $t$ is of order $\ln (V)$.

Beyond this time $t$, one approaches a stationary state with density $\rho$. At $\lambda=\lambda_{c}$, the dynamics is equivalent to the local system with a source of particles $h=p \rho$. Thus, we find that the density obeys $h=p A_{\rho} h^{1 / \delta_{h}}$, or $h=\left(p A_{\rho}\right)^{\delta_{h} /\left(\delta_{h}-1\right)}$ and thus

$$
\rho=A_{\rho}\left(p A_{\rho}\right)^{1 /\left(\delta_{h}-1\right)} .
$$

Eq. (14) should be compared to Eq. (7). It implies that the transition to a spreading epidemic happens at $\lambda=\tilde{\lambda}_{c}<\lambda_{c}$. For $\lambda \approx \tilde{\lambda}_{c}$, following the same steps as in the equilibrium case leads to the same result as Eq. (10), except that the role of temperature $T$ is replaced by $\lambda$, and the role of magnetization $m$ is replaced by density $\rho$. The transition is again mean-field. Thus, the stationary results in this non-equilibrium model are described by the same scaling theory as in the equilibrium models, while the spread of infection starting from a single source is described by an interesting branching dynamics.

Discussion - We have developed a general scaling theory for describing equilibrium and non-equilibrium system with both short and long-range interactions. We find that the long-range interactions lead to mean-field behavior, but with a scaling region whose width vanishes as $p \rightarrow 0$. We have also developed a branching process description of the spread of infection from a single source in the contact process with long-range interactions.

Acknowledgments - I thank Z. Toroczkai for discussion and $\mathrm{P}$. Fenimore for a careful reading. This work was supported by DOE W-7405-ENG-36.

[1] R. Albert and A.-L. Barabasi, Rev. Mod. Phys. 74, 47 (2002).

[2] D. J. Watts and S. H. Strogatz, Nature 393, 440 (1998).

[3] A. Barrat and M. Weigt, Eir. Phys. J. B. 13, 547 (2000).

[4] M. Gitterman, J. Phys. A 33, 8373 (2000).

[5] B. J. Kim et. al., Phys. Rev. E 64, 56135 (2001).

[6] C. P. Herrero, Phys. Rev. E 65, 66110 (2002).

[7] A. V. Goltsev, S. N. Dorogovtsev, and J. F. F. Mendes, Phys. Rev. E 67, 026123 (2003)

[8] M. Leone, A. Vazquez, A. Vespignani, and R. Zecchina, Eur. Phys. J. B 28, 191 (2002).

[9] M. Kardar, Phys. Rev. B 28, 244 (1983).

[10] M. E. J. Newman, C. Moore, and D. J. Watts, Phys. Rev. Lett. 84, 3201 (2000).

[11] A. B. Harris, J. Phys. C 7, 1671 (1974).

[12] A. A. Migdal, Sov. Phys. JETP 69, 810; L457 (1975); L. P. Kadanoff, Ann. Phys. (N. Y.) 100, 359 (1976).

[13] J. Zinn-Justin, Quantum Field Theory and Critical Phenomena, (Oxford Science Publications, 2001).

[14] T. E. Harris, Ann. Probab. 2, 969 (1974); J. Marro and R. Dickman, Nonequilibrium Phase Transitions in Lattice Models, (Cambridge University Press, 1999). 